\title{
STABILIZATION OF FUZZY SYSTEMS WITH CONSTRAINED CONTROLS BY USING POSITIVELY INVARIANT SETS
}

\author{
A. EL HAJJAJI, A. BENZAOUIA, AND M. NAIB \\ Received 11 June 2005; Revised 10 April 2006; Accepted 28 April 2006
}

We deal with the extension of the positive invariance approach to nonlinear systems modeled by Takagi-Sugeno fuzzy systems. The saturations on the control are taken into account during the design phase. Sufficient conditions of asymptotic stability are given ensuring at the same time that the control is always admissible inside the corresponding polyhedral set. Both a common Lyapunov function and piecewise Lyapunov function are used.

Copyright (c) 2006 A. El Hajjaji et al. This is an open access article distributed under the Creative Commons Attribution License, which permits unrestricted use, distribution, and reproduction in any medium, provided the original work is properly cited.

\section{Introduction}

Most plants in the industry have sever nonlinearity associated to saturations on the control. With the development of fuzzy systems, it is now possible to obtain a nonlinear representation by the qualitative knowledge of a system. On behalf of this idea, some fuzzy-model-based control system design methods have appeared in the fuzzy control field $[14,17,18]$ leading to many applications on nonlinear systems $[1,10]$. The nonlinear system is represented by a Takagi-Sugeno-(TS-) type fuzzy model. However, to the best of our knowledge, the nonquadratic saturations on the control are taken into account in the design of the fuzzy control only for a class of fuzzy systems [5].

It is well known that all these plants admit inputs limitation which are modeled by constraints of inequality type. The regulator problem for linear systems with constrained control was widely studied during these two decades. The tool of positive invariance was successfully applied to almost all the systems with constrained control, see, for example, [2-7] and the references therein.

In this paper, the saturations on the control are taken into account with the fuzzy model. The concept of positive invariance is used to obtain sufficient conditions of asymptotic stability for the global fuzzy system with constrained control inside a subset of the state space. The main idea of $[8,9]$ representing the nonlinear system by a set of

Hindawi Publishing Corporation

Mathematical Problems in Engineering

Volume 2006, Article ID 13832, Pages 1-17

DOI 10.1155/MPE/2006/13832 
uncertain-like linear subsystems is used in this paper. The problem is then to design a controller which is "robust" with respect to the upper bound extreme subsystems by taking into account the saturations on the control. Both a common Lyapunov function and a piecewise Lyapunov function as used in $[8,9,13]$ are used to analyze and design the controllers which ensure asymptotic stability of the nonlinear system despite the presence of saturations on the control. Hence, a set of linear matrix inequalities (LMIs) is proposed to build stabilizing controllers together with their corresponding region of asymptotic stability and positive invariance.

This paper is organized as follows: Section 2 deals with the problem presentation while Section 3 presents some preliminary results. The main results of the paper are given by Section 4. An example is studied in Section 5.

\section{Notation and problem presentation}

The notations used in this paper are as follows.

(i) For two vectors $x, y \in \mathbb{R}^{n}, x \preceq y$ if $x_{i} \preceq y_{i}, i=1, \ldots, n$.

(ii) For a matrix $H \in \mathbb{R}^{n \times n}, h_{i j}^{+}=\operatorname{Sup}\left(h_{i j}, 0\right), h_{i j}^{-}=\operatorname{Sup}\left(-h_{i j}, 0\right)$, for $i, j=1, \ldots, n$, where $h_{i j}$ denotes the matrix component $H(i, j)$.

(iii) $\hat{H}(i, i)=H(i, i) ; \hat{H}(i, j)=|H(i, j)|, i \neq j, i, j=1, \ldots, n$, where $|H(i, j)|$ denotes the module of the matrix component $H_{i j}$.

(iv) $|H|(i, j)=|H(i, j)|, i, j=1, \ldots, n$.

(v) For a positive definite matrix $P, P>0$.

Consider the following nonlinear system with constrained control that can be described by the TS fuzzy model if $x_{1}(t)$ is $M_{i 1}$ and ... and $x_{n}(t)$ is $M_{\text {in }}$ then

$$
\dot{x}(t)=A_{i} x(t)+B_{i} u(t), \quad i=1, \ldots, r,
$$

where $M_{i j}$ is the fuzzy set, $r$ is the number of if-then rules, $x \in \mathbb{R}^{n}$ is the state, and $u \in \mathbb{R}^{m}$ is the control which is constrained as follows:

$$
u \in \Omega=\left\{u \in \mathbb{R}^{m},-q_{2} \preceq u \preceq q_{1} ; q_{1}, q_{2} \in \mathbb{R}^{m}\right\} .
$$

Following [15-18], the global model is structured in the following form:

$$
\dot{x}(t)=A(\alpha) x(t)+B(\alpha) u(t)
$$

with

$$
\begin{gathered}
\alpha=\left[\alpha_{1} \cdots \alpha_{r}\right]^{\top} \\
A(\alpha)=\sum_{i=1}^{r} \alpha_{i}(x(t)) A_{i} ; \quad B(\alpha)=\sum_{i=1}^{r} \alpha_{i}(x(t)) B_{i} ;
\end{gathered}
$$


where $\alpha_{i}(x(t))$ is the normalized membership function of the inferred fuzzy set $M_{i}=$ $\prod_{l=1}^{n} M_{i l}$ and

$$
\alpha_{i}(x(t)) \geq 0, i=1, \ldots, r ; \quad \sum_{i=1}^{r} \alpha_{i}(x(t))=1
$$

Let $M$ be the set of membership functions satisfying (2.5).

Matrices $A_{i}$ and $B_{i}$ are constant of appropriate size and satisfy the following assumptions:

(H) each pair $\left(A_{i}, B_{i}\right)$ is stabilizable.

In general, the control is given by

$$
u(t)=F(\alpha) x(t)
$$

This control leads to the following system in closed loop:

$$
\dot{x}(t)=[A(\alpha)+B(\alpha) F(\alpha)] x(t)
$$

The main objective of this paper is to design the controller $F(\alpha)$ such that the global system is asymptotically stable at the origin despite the presence of constraints on the control. To achieve this objective, two techniques will be used. The first consists in rewriting equivalently the initial system (2.1) by using a state space repartition allowing to introduce $r$-like uncertain subsystems as used before by many authors. The second concerns the use of the so-called positive invariance approach which will enable one to construct regions of linear behavior for the system with saturations on the control.

\section{Preliminary results}

In this section, we recall the approach of positive invariance as known in the literature applied to a linear time-invariant system. Consider the following system given by

$$
\dot{x}(t)=A x(t)
$$

Let the state be constrained as follows:

$$
\mathscr{D}=\left\{x \in \mathbb{R}^{n} \mid-\delta_{2} \preceq x \preceq \delta_{1} ; \delta_{1}, \delta_{2} \in \mathbb{R}^{n}\right\} .
$$

We recall here the approach proposed in $[3,4,6]$.

Definition 3.1. A subset $\mathscr{D}$ of $\mathbb{R}^{n}$ is said to be positively invariant with respect to the motion of the system (3.1) if for every initial state $x_{o} \in \mathscr{D}$, the motion $x\left(x_{0}, t\right) \in \mathscr{D}$, for every $t$.

The necessary and sufficient condition of domain $\mathscr{D}$ to be positively invariant with respect to system $(3.1)$ is given by $[3,6]$. 
$4 \quad$ Fuzzy systems with constrained control

THEOREM 3.2. The set $\mathscr{D}$ is positively invariant with respect to system (3.1) if and only if

$$
\widetilde{A} \delta \preceq 0,
$$

where

$$
\tilde{A}=\left[\begin{array}{cc}
A_{1} & A_{2} \\
A_{2} & A_{1}
\end{array}\right] ; \quad A_{1}=\left\{\begin{array}{l}
a_{i i} \\
a_{i j}^{+}
\end{array} \quad \text { for } i \neq j ; \quad A_{2}=\left\{\begin{array}{l}
0 \\
a_{i j}^{-}
\end{array} \quad \text { for } i \neq j ; \quad \delta=\left[\begin{array}{l}
\delta_{1} \\
\delta_{2}
\end{array}\right] .\right.\right.
$$

Remark 3.3. If the constraints are symmetric, that is, $\delta_{1}=\delta_{2}$, the condition of positive invariance of the set $\mathscr{D}$ with respect to the system (3.1) becomes

$$
\widehat{A} \delta_{1} \preceq 0,
$$

where matrix $\hat{A}$ is given by

$$
\widehat{A}=\left\{\begin{array}{l}
a_{i i} \\
\left|a_{i j}\right|
\end{array} \quad \text { for } i \neq j .\right.
$$

Note that $A=A_{1}-A_{2} ;|A|=A_{1}+A_{2}$.

\section{Main results}

In this section, we propose sufficient conditions of asymptotic stability for the system with constrained control by using both common Lyapunov and piecewise Lyapunov functions. These results are based on the technique of rewriting equivalently the fuzzy system (2.3) under the form of $r$-like uncertainty subsystems as proposed in $[8,9]$.

Consider the nonlinear system with constrained control that can be described by the TS fuzzy model (2.1). Following the idea of $[8,9]$, one can divide the input space into fuzzy subspaces and build a linear model, called the local model, in each subspace. Then, the membership function is used to connect smoothly the local models together to form a global fuzzy model of the nonlinear system. Define the $r$ subspaces in the state space as follows:

$$
S_{j}=\left\{x \mid \alpha_{j}(x) \geq \alpha_{i}(x), i=1,2, \ldots, r, i \neq j\right\}, \quad j=1,2, \ldots, r .
$$

The characteristic function of $S_{j}$ is defined by

$$
\eta_{j}= \begin{cases}1, & x \in S_{j} ; \\ 0, & x \notin S_{j} ; \quad \sum_{j=1}^{r} \eta_{j}=1\end{cases}
$$

See Figure 5.1 for the example of a repartition of the state space on two subspaces $S_{1}$ and $S_{2}$ related to the corresponding membership function. 
A. El Hajjaji et al. 5

On every subspace $S_{j}$ the fuzzy system can be denoted by

$$
\dot{x}(t)=\left(A_{j}+\Delta A_{j}(t)\right) x(t)+\left(B_{j}+\Delta B_{j}(t)\right) u(t)
$$

with

$$
\Delta A_{j}(t)=\sum_{i=1, i \neq j}^{r} \alpha_{i}(t)\left(A_{i}-A_{j}\right) ; \quad \Delta B_{j}(t)=\sum_{i=1, i \neq j}^{r} \alpha_{i}(t)\left(B_{i}-B_{j}\right) .
$$

It is assumed that if the $j$ th subsystem is in the $j$ th subspace, it will stay in this subspace for a time $t_{j}>\tau, \tau>0$ is a fixed constant. The number of traversing time instants among the regions is also assumed to be finite.

Remark 4.1. It is useful to note that $\Delta A_{j}(t)$ and $\Delta B_{j}(t)$ are known at any time and the studied system is not an uncertain system. However, in order to obtain simpler stability conditions, this technique assumes that the terms $\Delta A_{j}(t)$ and $\Delta B_{j}(t)$ are like uncertain terms and are bounded.

Following the idea of $[8,9]$, we assume that an upper bound of each uncertain-like term is known and is given by

$$
\begin{aligned}
& -E_{1 j} \leq \Delta A_{j}(t) \leq E_{1 j}, \quad E_{1 j} \geq 0, \forall t \geq 0, j=1, \ldots, r, \\
& -E_{2 j} \leq \Delta B_{j}(t) \leq E_{2 j}, \quad E_{2 j} \geq 0, \forall t \geq 0, j=1, \ldots, r .
\end{aligned}
$$

This type of inequality bounds can always be transformed to the following quadratic bounds:

$$
\begin{array}{ll}
{\left[\Delta A_{j}(t)\right]^{T}\left[\Delta A_{j}(t)\right] \leq E_{1 j}^{T} E_{1 j}} & \forall t \succeq 0 ; j=1, \ldots, r, \\
{\left[\Delta B_{j}(t)\right]^{T}\left[\Delta B_{j}(t)\right] \leq E_{2 j}^{T} E_{2 j} \quad \forall t \succeq 0 ; j=1, \ldots, r .}
\end{array}
$$

Note that the details about the estimation of the upper bounds according to (4.5) are widely developed in $[8,9]$. We obtain $r$ distinct linear time-varying subsystems. The stabilization problem of the fuzzy system (2.1) without saturation constraints has been studied in $[8,9]$ by using extreme subsystems obtained with the upper bounds of the uncertainty-like terms (4.5). In our case, the upper uncertainty bounds are also used to obtain asymptotic stability conditions, while, the uncertain-like subsystems are used directly to built necessary and sufficient conditions of positive invariance.

The control is constrained as follows:

$$
u \in \Omega=\left\{u \in \mathbb{R}^{m},-q_{2} \preceq u \preceq q_{1} ; q_{1}, q_{2} \in \mathbb{R}^{m}\right\} .
$$

The idea of this approach is to choose on every subspace $S_{j}, j \in 1, \ldots, r$, the fuzzy subsystem (4.3) and consider that the interaction of the corresponding system with all the remainder $r-1$ subsystems is taken into account by the uncertainty-like terms $\Delta A_{j}(t)$ and $\Delta B_{j}(t)$. The objective is then to design for such a subsystem a feedback control given 
by

$$
u(t)=F_{j} x(t), \quad x(t) \in S_{j},
$$

which guarantees the asymptotic stability of the uncertain-like subsystems (4.3) despite the presence of the saturations (4.7). The subsystem in closed loop is given by

$$
\dot{x}(t)=\left[\left(A_{j}+B_{j} F_{j}\right)+\left(\Delta A_{j}(t)+\Delta B_{j}(t) F_{j}\right)\right] x(t) .
$$

Note that the control in system (2.3) can be considered in this approach as a switching control formed by all the subsystems controls and given by

$$
u(t)=\sum_{j=1}^{r} \eta_{j} F_{j} x(t) .
$$

In the constrained case, we follow the approach proposed in $[3,4,6]$. Recall that this approach consists in giving conditions that allow the choice of a stabilizing controller (4.8) in such a way that model (4.9) remains valid every time. This is only possible if the state is constrained to evolve in a specified region defined by

$$
\mathscr{D}_{j}=\left\{x \in \mathbb{R}^{n} \mid-q_{2} \preceq F_{j} x \preceq q_{1} ; q_{1}, q_{2} \in \mathbb{R}^{m}\right\} .
$$

Note that these domains are convex and unbounded for $m<n$.

The result of stabilizability of the fuzzy system without constrained control, using the idea of $[8,9]$ based on the upper extreme subsystems to obtain conditions of asymptotic stability for the fuzzy system (2.3), is recalled below according to the following definition.

Definition 4.2. The system (2.3) is said to be quadratically stabilizable if there exist a control law (2.6), a positive symmetric matrix $P$, and a scalar $\gamma>0$ such that the following condition is satisfied:

$$
\dot{V}(x(t))=x(t)^{T}\left\{[A(\alpha)+B(\alpha) F(\alpha)]^{T} P+P[A(\alpha)+B(\alpha) F(\alpha)]\right\} x(t) \leq-\gamma\|x\|^{2}
$$

for all $x(t) \in \mathbb{R}^{n}$, for all $\alpha \in \mathcal{M}$, for all $t>0$, where $V(x)=x^{T} P x$ is a Lyapunov function.

It is worth noting that if the system (2.3) is quadratically stabilizable, then function $V(x)$ is a Lyapunov function for the closed-loop system (2.7). Then, the equilibrium point $x=0$ will be uniformly asymptotically stable at large.

Leмma $4.3[8,9]$. The fuzzy system (2.3) is quadratically stabilizable if and only if there exists a set of feedback gains $\left(F_{1}, F_{2}, \ldots, F_{r}\right)$ such that the following closed-loop subsystems with the accurate upper bounds are quadratically stable:

$$
\dot{x}(t)=\left(A_{j}+E_{j 1}\right) x(t)+\left(B_{j}+E_{j 2}\right) F_{j} x(t), \quad x(t) \in S_{j}, j=1, \ldots, r .
$$


Recall that the stability result obtained by $[8,9]$ is based on the use of Lemma 4.3 and a piecewise Lyapunov function candidate, as used by [13], given by

$$
V(x)=x^{T}\left(\sum_{j=1}^{r} \eta_{j} P_{j}\right) x(t) .
$$

In our case, we first consider a common Lyapunov function for the application of Lemma 4.3, that is, $P_{1}=\cdots=P_{r}$. In this case, function (4.14) becomes $V(x)=x^{T} P x$. Define its level set by the following:

$$
\Psi(P, \rho)=\left\{x \mid x^{T} P x \preceq \rho, \rho \succ 0\right\} .
$$

The use of the Lemma 4.3 and the result of [11] enable us to state the main result of this paper concerning the asymptotic stability of the fuzzy system (2.3) with the saturations $(2.2)$.

Theorem 4.4. If there exist a symmetric positive definite matrix $P \in \mathbb{R}^{n x n}$ and a positive scalar $\rho$ such that

$$
\begin{gathered}
\left(A_{j}+B_{j} F_{j}\right)^{T} P+P\left(A_{j}+B_{j} F_{j}\right)+\left(E_{j 1}+E_{j 2} F_{j}\right)^{T} P+P\left(E_{j 1}+E_{j 2} F_{j}\right)<0 ; \quad j=1, \ldots, r ; \\
\Psi(P, \rho) \subset \mathscr{D}_{j}, \quad j=1, \ldots, r
\end{gathered}
$$

then, the fuzzy system (2.3) with the feedback control (4.10) is asymptotically stable for all $x_{0} \in \Psi(P, \rho)$.

Proof. Conditions (4.16) imply that the function $V(x)=x^{T} P x$ is a common Lyapunov function of all the upper bound extreme subsystems (4.13). Recall that the level set $\Psi(P, \rho)$ of the common Lyapunov function is positively invariant with respect to the upper bound extreme subsystems. According to Lemma 4.3 and Definition 4.2, this set is also a level set (region of stability) for the uncertain-like subsystems (4.9), that is, the set $\Psi(P, \rho)$ is also positively invariant with respect to the uncertain-like subsystems (4.9). Thus, the control is always admissible, that is, $-q_{2} \preceq F_{j} x(t) \preceq q_{1}$, for all $t \succeq 0$ by virtue of conditions (4.17). Consequently, each control $u(t)=F_{j} x(t)$ is admissible for all $x_{0} \in \Psi(P, \rho)$ and the linear subsystem (4.9) is always valid inside this region of linear behavior. Hence, it is obvious that by applying the switching control (4.10) to the uncertain-like fuzzy system (4.3), the control remains admissible by virtue of the following:

$$
-q_{2} \preceq F_{j} x(t) \preceq q_{1} \quad \forall t \succeq 0 \quad \text { implies } \quad-q_{2} \preceq \sum_{j=1}^{r} \eta_{j} F_{j} x(t) \preceq q_{1} \quad \forall t \succeq 0, j=1, \ldots, r,
$$

where $\eta$ is defined by (4.2). In order to guarantee that this implication remains satisfied even if the state switches from a subspace $S_{j}$ to a different subspace $S_{i}, i \neq j$, it is necessary to take the initial state inside the common domain $\Psi(P, \rho)$. The positive invariance property of the set $\Psi(P, \rho)$ implies that all the uncertain subsystems (4.9) remain linear 
despite the presence of the saturations. This fact allows the application of the Lemma 4.3 and Definition 4.2 to these uncertain-like subsystems to obtain $r$ upper bound extreme subsystems by using the assumptions (4.4). If in addition the feedback controllers $F_{j}$ satisfy conditions (4.16), then the global fuzzy system (2.3) with the feedback control (4.10) is asymptotically stable at the origin for all $x_{0} \in \Psi(P, \rho)$ despite the presence of saturations.

Note that a different condition than (4.16) was presented by $[8,9]$ based on the wellknown separation lemma

$$
X^{T} Y+Y^{T} X \leq \varepsilon X^{T} X+\frac{1}{\varepsilon} Y^{T} Y
$$

for any positive scalar $\varepsilon$ and matrices $X, Y$. In our case, condition (4.16) is easily resolved by LMI technique.

It is worth noting that to include a symmetric ellipsoid inside a nonsymmetrical polyhedra, it is sufficient to realize this only inside the symmetrical part of the polyhedra. This means in our case, to realize (4.17) only with $q=\min \left(q_{1}, q_{2}\right)$. It is well known that to obtain condition (4.17), one has only to satisfy the following inequalities [12]:

$$
\rho f_{j}^{i} P^{-1}\left(f_{j}^{i}\right)^{T} \preceq q_{i}^{2}, \quad j=1, \ldots, r, i=1, \ldots, m,
$$

where $f_{j}^{i}$ is the $i$ th row of matrix $F_{j}, q=\min \left(q_{1}, q_{2}\right)$. These inequalities can be transformed by the use of Schur complement to the following LMI:

$$
\left[\begin{array}{ll}
\mu_{i} & y_{j}^{i} \\
* & X
\end{array}\right] \geq 0, \quad i=1, \ldots, m,
$$

where $y_{j}^{i}$ is the $i$ th row of matrix $Y_{j}=F_{j} X, X=P^{-1}$, and $\mu_{i}=q_{i}^{2} / \rho$.

The result of Theorem 4.4 is now used for the control synthesis.

THeOREM 4.5. If there exist a symmetric matrix $X, r$ matrices $Y_{1}, \ldots, Y_{r}$, and positive scalar $\rho$ solutions of the following LMIs:

$$
\begin{gathered}
X\left(A_{j}+E_{1 j}\right)^{T}+Y_{j}^{T}\left(B_{j}+E_{2 j}\right)^{T}+\left(A_{j}+E_{1 j}\right) X+\left(B_{j}+E_{2 j}\right) Y_{j}<0, \\
{\left[\begin{array}{cc}
\mu_{i} & y_{j}^{i} \\
* & X
\end{array}\right] \geq 0,} \\
X>0, \quad j=1, \ldots, r, \quad i=1, \ldots, m,
\end{gathered}
$$

where $\mu_{i}=q_{i}^{2} / \rho, y_{j}^{i}$ is the ith row of matrix $Y_{j}$, then, the fuzzy system (2.3) with the feedback control (4.10) with

$$
F_{j}=Y_{j} X^{-1}, \quad P=X^{-1}
$$

is asymptotically stable at the origin for all $x_{0} \in \Psi(P, \rho)$. 
Proof. Follows readily from Theorem 4.4 .

This result is easily applied to design controllers: solving the LMIs (4.22) by any common available software (in our case we used the matlab LMI control toolbox), matrix $P$ and the controller gains $F_{i}$ can be computed easily according to the equalities (4.23). Nevertheless, a common Lyapunov function for all the $r$ upper bound extreme subsystems does not always exist. We can then attempt to use a piecewise Lyapunov function candidate as used by $[8,9]$. The use of this type of function is not easy when the system is, in addition, with constrained control. The following result proposes a sufficient condition of asymptotic stability based on a piecewise function.

Define the following polyhedral set:

$$
\Gamma(\delta)=\{x \mid-\delta \preceq x \preceq \delta ; \delta \succ 0\}
$$

In this approach, we would like to design all the controller gains $F_{j}$ such that all the level sets associated to matrices $P_{j}, j=1, \ldots, r$, contain the same polyhedra $\Gamma(\delta)$. This is possible if we add the following constraint to our problem:

$$
\Gamma(\delta) \subset \Psi\left(P_{j}, \rho_{j}\right), \quad j=1, \ldots, r .
$$

Remark 4.6. Condition (4.25) can also be given in LMI form. For this, redefine the polyhedral set $\Gamma(\delta)$ in the equivalent form

$$
\Gamma(\delta)=\operatorname{cov}\left\{v_{1}, v_{2}, \ldots, v_{k}\right\}
$$

where $v_{l} \in \mathbb{R}^{n}$ denotes the vertex of the bounded polyhedron $\Gamma(\delta)$. Note that, $k=2^{n}$. With this, condition (4.25) is equivalent to

$$
v_{l}^{T} P_{j} v_{l} \leq \rho_{j}, \quad l=1, \ldots, k, j=1, \ldots, r .
$$

By virtue of Schur complement, this latter is equivalent to

$$
\left[\begin{array}{ll}
\rho_{j} & v_{l}^{T} \\
* & X_{j}
\end{array}\right] \geq 0, \quad j=1, \ldots, r, l=1, \ldots, k
$$

with $X_{j}=P_{j}^{-1}$

The following result ensures the realization of this objective.

Theorem 4.7. For given positive scalars $\rho_{1}, \ldots, \rho_{r}$ and positive vector $\delta$, if there exist symmetric definite positive matrices $X_{1}, \ldots, X_{r}$ and matrices $Y_{1}, \ldots, Y_{r}$, solutions of the following 
LMIs:

$$
\begin{gathered}
X_{j}\left(A_{j}+E_{1 j}\right)^{T}+Y_{j}^{T}\left(B_{j}+E_{2 j}\right)^{T}+\left(A_{j}+E_{1 j}\right) X_{j}+\left(B_{j}+E_{2 j}\right) Y_{j}<0, \\
{\left[\begin{array}{cc}
\frac{q_{i}^{2}}{\rho_{j}} & y_{j}^{i} \\
* & X_{j}
\end{array}\right] \geq 0,} \\
{\left[\begin{array}{cc}
\rho_{j} & v_{l}^{T} \\
* & X_{j}
\end{array}\right] \geq 0, \quad X_{j}>0, j=1, \ldots, r, i=1, \ldots, m, l=1, \ldots, k,}
\end{gathered}
$$

such that the matrices in closed loop satisfy

$$
\widehat{A}_{c j} \delta+\left|E_{c j}\right| \delta \preceq 0,
$$

where $A_{c j}=A_{j}+B_{j} Y_{j} X_{j}^{-1}, E_{c j}=E_{1 j}+E_{2 j} Y_{j} X_{j}^{-1}$, and $v_{1}, \ldots, v_{k}$ are the corresponding vertices to vector $\delta$, then, the fuzzy system (2.3) with the feedback control (4.10) is asymptotically stable at the origin for all $x_{0} \in \Gamma(\delta)$.

Proof. Based on Lemma 4.3 and the use of the piecewise Lyapunov function candidate (4.14), the feasibility of the LMIs (4.29)-(4.31) gives symmetric positive definite matrices $P_{j}=X_{j}^{-1}$ and gain controllers $F_{j}=Y_{j} X_{j}^{-1}$, thus ensuring the asymptotic stability at the origin of the fuzzy system (2.3) with the feedback control (4.10) which is always admissible by virtue of conditions (4.30), that is, each level set $\Psi\left(P_{j}, \rho_{j}\right) \subset \mathscr{D}_{j}$. Note also, that all the obtained level sets $\Psi\left(P_{j}, \rho_{j}\right) \supset \Gamma(\delta)$. The new problem in this approach with a piecewise function and a switching control, is, even if inside any level set $\Psi\left(P_{j}, \rho_{j}\right)$, the control is admissible, this property may be lost when a switch occurs according to the strategy (4.2). This problem can be solved if we can ensure for the system that the common set $\Gamma(\delta)$ is positively invariant with respect to all the $r$ uncertain subsystems (4.3). This fact is realized with condition (4.32), which is a direct application of Theorem 3.2. In this case, the state of the system belongs inside all the sets $\mathscr{D}_{j}$, that is, the state feedback control is always admissible, for all $x_{0} \in \Gamma(\delta)$.

The result of this theorem can be used in two steps: the first step consists in computing the solutions of the LMIs (4.29)-(4.31). With these solutions, the matrices in closed loop are computed. The second step consists in testing conditions (4.32) with vector $\delta$ as a parameter of design.

\section{Example}

In this section, we apply our results to two examples. The first is the one studied in $[8,9]$. Consider the problem of balancing an inverted pendulum on a cart. The equations of the motion for the pendulum are

$$
\begin{gathered}
\dot{x}_{1}=x_{2}, \\
\dot{x}_{2}=\frac{g \sin \left(x_{1}\right)-\operatorname{aml} x_{2}^{2} \sin \left(2 x_{1}\right) / 2-a \cos \left(x_{1}\right) u}{4 l / 3-\operatorname{aml} \cos ^{2}\left(x_{1}\right)},
\end{gathered}
$$


A. El Hajjaji et al. 11

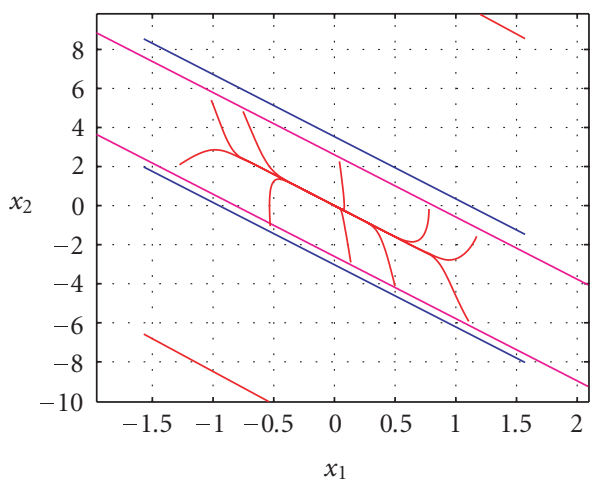

(a)

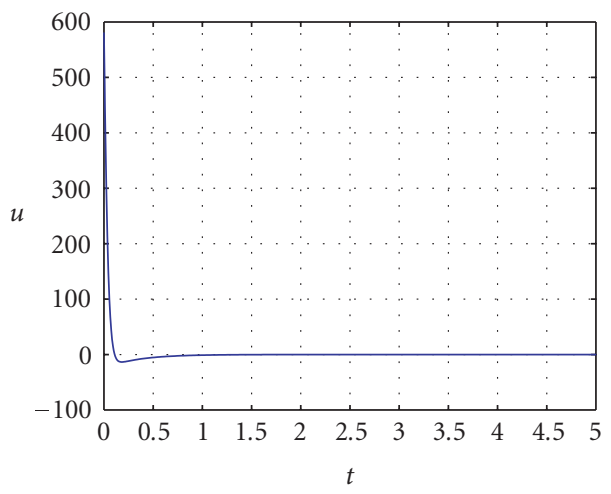

(b)

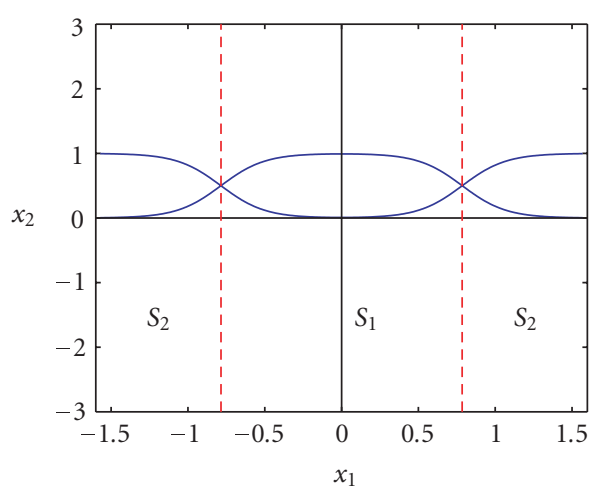

(c)

Figure 5.1. This figure presents in red color the evolution of the state of the system in closed loop inside the set of asymptotic stability $\Psi(P, \rho)$ (in magneta color) for different initial states, the evolution of the control, and the membership function together with the sets $S_{1}$ and $S_{2}$.

where $x_{1}$ denotes the angle of the pendulum from the vertical, and $x_{2}$ is the angular velocity. $g$ is the gravity acceleration, $m$ the mass of the pendulum, $M$ is the mass of the cart, $2 l$ is the length of the pendulum, and $u$ is the force applied to the cart. $a=1 /(m+M)$. The following data are chosen: $m=2 \mathrm{~kg}, M=8 \mathrm{~kg}$, and $2 l=1$ meter. We also add the following saturation on the control:

$$
-3000 \preceq u \preceq 3500 .
$$

The following fuzzy model is used to design a fuzzy controller.

(i) Rule 1: if $x_{1}(t)$ is about 0 , then $\dot{x}(t)=A_{1} x(t)+B_{1} u(t)$.

(ii) Rule 2: if $x_{1}(t)$ is about $\pm \pi / 2$, then $\dot{x}(t)=A_{2} x(t)+B_{2} u(t)$, 
with

$$
\begin{gathered}
A_{1}=\left[\begin{array}{cc}
0 & 1 \\
17.2941 & 0
\end{array}\right] ; \quad A_{2}=\left[\begin{array}{cc}
0 & 1 \\
9.3648 & 0 .
\end{array}\right] ; \\
B_{1}=\left[\begin{array}{c}
0 \\
-0.1765
\end{array}\right] ; \quad B_{2}=\left[\begin{array}{c}
0 \\
-0.0054
\end{array}\right] .
\end{gathered}
$$

The membership functions and the upper bounds as used by $[8,9]$ are as follows:

$$
\begin{gathered}
M_{1}^{1}\left(x_{1}(t)\right)=\left(1-\frac{1}{1+\exp \left[-7\left(x_{1}(t)-\pi / 4\right)\right]}\right) \frac{1}{1+\exp \left[-7\left(x_{1}(t)+\pi / 4\right)\right]}=\alpha_{1}(t), \\
M_{1}^{2}\left(x_{1}(t)\right)=1-M_{1}^{1}\left(x_{1}(t)\right)=\alpha_{2}(t) \\
E_{11}=E_{12}=0.1\left|A_{1}-A_{2}\right| ; \quad E_{21}=E_{22}=0.01\left|B_{1}-B_{2}\right| .
\end{gathered}
$$

By applying Theorem 4.5, we find the following result:

$$
P=\left[\begin{array}{ll}
14.9944 & 4.7034 \\
4.7034 & 1.4756
\end{array}\right] \text {. }
$$

The obtained gain controllers are given by

$$
F_{1}=\left[\begin{array}{ll}
849.7047 & 253.0999
\end{array}\right] ; \quad F_{2}=[3156.8990 .5]
$$

The common set $\Psi(P, \rho=10)$ of asymptotic stability is given by Figure 5.1 together with the sets $\mathscr{D}_{j}$.

The application of Theorem 4.7 leads to nonfeasible LMIs due to the structure of matrices $A_{1}, A_{2}, B_{1}, B_{2}$ which are under Compagnon form. With any feedback controller, the matrices of the system in closed loop remain under the same form. Hence, condition (4.32) cannot be satisfied. To overcome this problem, one has to apply any nonsingular transformation to the initial linear subsystems.

Consider now the following constrained nonlinear system:

$$
\begin{gathered}
\dot{x}_{1}(t)=-2.1 x_{1}+1.5 x_{2}(t)+2.5 u_{1}(t)+0.5 u_{2}(t), \\
\dot{x}_{2}(t)=3.5 x_{1}(t)-0.5\left[0.5+\ln \left(x_{1}^{2}+1\right)\right] x_{2}(t)+u_{1}(t)-1.5 u_{2}(t),
\end{gathered}
$$


where the control is constrained as follows:

$$
-q_{2} \preceq u \preceq q_{1} ; \quad q_{1}=\left[\begin{array}{l}
35 \\
45
\end{array}\right] ; q_{2}=\left[\begin{array}{l}
40 \\
45
\end{array}\right] .
$$

Now we give the exact approximation of the nonlinear system by a TS model. For this, assume that $x_{1}(t) \in[-\gamma, \gamma]$, then one can write

$$
\ln \left(x_{1}^{2}+1\right)=M_{1}^{1}\left(x_{1}(t)\right) \cdot 0+M_{1}^{2}\left(x_{1}(t)\right) \cdot \ln \left(\gamma^{2}+1\right)
$$

with

$$
\begin{gathered}
M_{1}^{1}\left(x_{1}(t)\right)=\frac{\ln \left(\gamma^{2}+1\right)-\ln \left(x_{1}^{2}+1\right)}{\ln \left(\gamma^{2}+1\right)}=\alpha_{1}(t), \\
M_{1}^{2}\left(x_{1}(t)\right)=1-M_{1}^{1}\left(x_{1}(t)\right)=\frac{\ln \left(x_{1}^{2}+1\right)}{\ln \left(\gamma^{2}+1\right)}=\alpha_{2}(t) .
\end{gathered}
$$

The fuzzy model which represents exactly the nonlinear system is given by

$$
\begin{aligned}
& \text { if } x_{1}(t) \text { is } M_{1}^{1} \text {, then } \dot{x}(t)=A_{1} x(t)+B_{1} u(t) ; \quad-q_{2} \preceq u \preceq q_{1}, \\
& \text { if } x_{1}(t) \text { is } M_{1}^{2} \text {, then } \dot{x}(t)=A_{2} x(t)+B_{2} u(t) ; \quad-q_{2} \preceq u \preceq q_{1},
\end{aligned}
$$

where matrices $A_{1}, A_{2}, B_{1}$, and $B_{2}$ are given by

$$
\begin{gathered}
A_{1}=\left[\begin{array}{cc}
-2.1 & 1.5 \\
3.5 & -0.25
\end{array}\right] ; \quad A_{2}=\left[\begin{array}{cc}
-2.1 & 1.5 \\
3.5 & -0.5\left(0.5+\ln \left(\gamma^{2}+1\right)\right)
\end{array}\right] ; \\
B_{1}=B_{2}=\left[\begin{array}{cc}
2.5 & 0.5 \\
1 & -1.5
\end{array}\right] .
\end{gathered}
$$

For this fuzzy system composed of two subsystems, one can take the following upper bounds:

$$
E_{11}=0.5\left|A_{2}-A_{1}\right| ; \quad E_{21}=0 ; \quad E_{12}=0.5\left|A_{1}-A_{2}\right| ; \quad E_{22}=0 .
$$

Choose $\gamma=12$.

The asymptotic stability is guaranteed by the existence of one symmetric positive definite matrix by resolving the LMI (4.22):

$$
P=\left[\begin{array}{cc}
0.0224 & -0.0016 \\
-0.0016 & 0.0127
\end{array}\right] \text {. }
$$




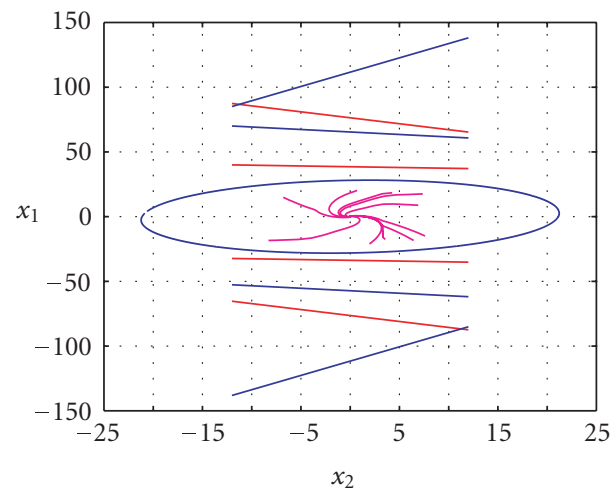

(a)

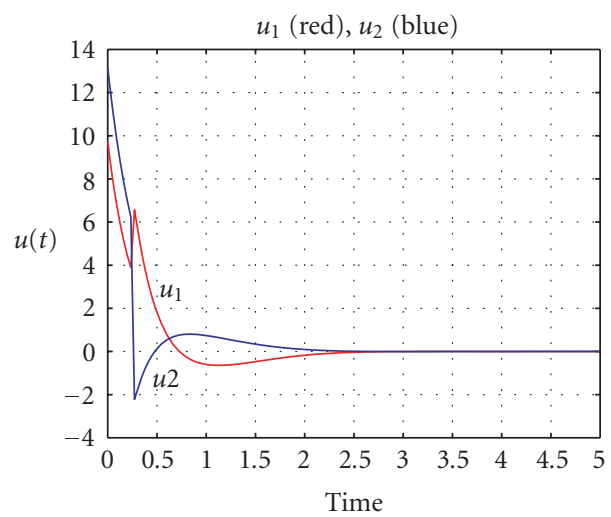

(b)

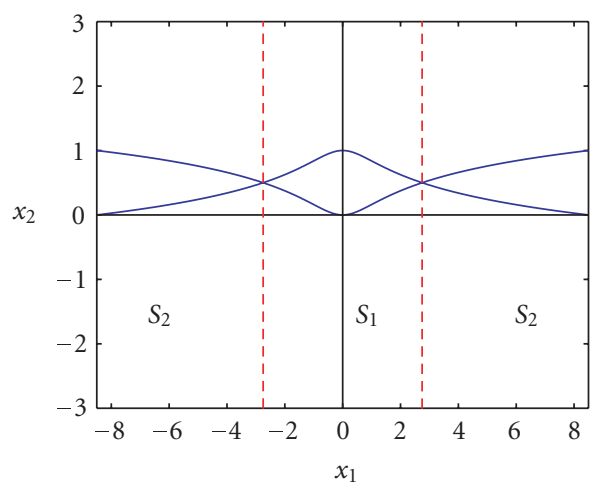

(c)

Figure 5.2. This figure presents the evolution of the state of the system in closed loop inside the set (in blue) of asymptotic stability $\Psi(P, \rho)$ for different initial states, the evolution of the control for an initial state inside $\Psi(P, \rho)$, and the membership function together with the sets $S_{1}$ and $S_{2}$.

The obtained gain controllers are given by

$$
F_{1}=\left[\begin{array}{cc}
-0.1243 & -1.0373 \\
0.5409 & 0.5891
\end{array}\right] ; \quad F_{2}=\left[\begin{array}{cc}
-0.2354 & -0.6114 \\
0.8910 & -0.4031
\end{array}\right]
$$

The common set $\Psi(P, \rho=10)$ of asymptotic stability is given by Figure 5.2 together with the sets $\mathscr{D}_{j}$.

Now, we apply the results of Theorem 4.7. The resolution of the LMIs (4.29)-(4.31) leads to the following solutions only for a reduced upper bound and interval of evolution 
A. El Hajjaji et al. 15

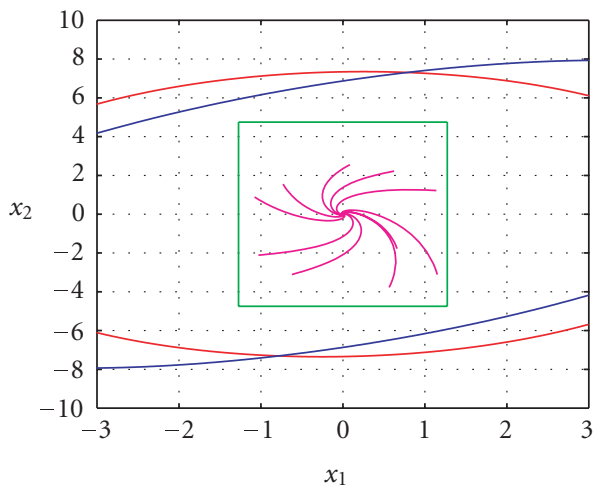

(a)

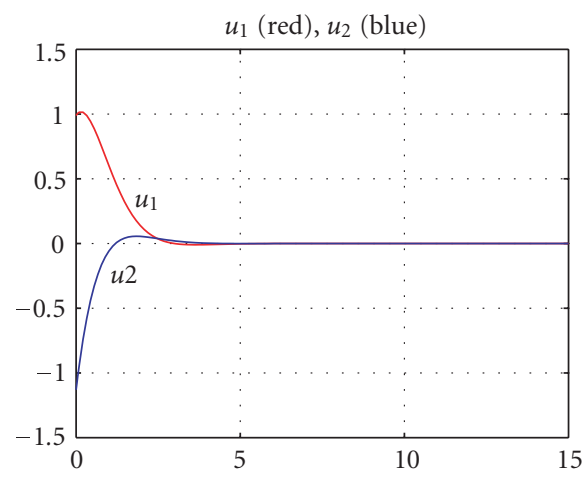

(b)

Figure 5.3. This figure presents the evolution of the state of the system in closed loop inside the common set of positive invariance $\Gamma(\delta)$ for different initial states and he evolution of the control for an initial state inside the common set of positive invariance $\Gamma(\delta)$.

of $x_{1}$ :

$$
\begin{gathered}
E_{11}=0.25\left|A_{2}-A_{1}\right| ; \quad E_{21}=0 ; \quad E_{12}=0.25\left|A_{1}-A_{2}\right| ; \quad E_{22}=0 ; \quad \gamma=8.5, \\
P_{1}=\left[\begin{array}{cc}
0.0141 & -0.0005 \\
-0.0005 & 0.0066
\end{array}\right] ; \quad P_{2}=\left[\begin{array}{cc}
0.0132 & -0.0053 \\
-0.0053 & 0.0085
\end{array}\right] .
\end{gathered}
$$

The obtained gain controllers are given by

$$
F_{1}=\left[\begin{array}{cc}
0.0184 & -0.7839 \\
0.9094 & 0.0645
\end{array}\right] ; \quad F_{2}=\left[\begin{array}{cc}
0.2772 & -0.5679 \\
0.8088 & -0.4785
\end{array}\right] \text {. }
$$

For a given polyhedral set $\Gamma(\delta)$, with $\delta=\left[\begin{array}{ll}1.2735 & 4.7475\end{array}\right]^{T}$, the condition (4.32) is also satisfied:

$$
\left(\hat{A}_{c 1}+\left|E_{c 1}\right|\right) \delta=\left[\begin{array}{l}
-0.0070 \\
-0.0761
\end{array}\right] ; \quad\left(\hat{A}_{c 2}+\left|E_{c 2}\right|\right) \delta=\left[\begin{array}{l}
-0.5216 \\
-4.8546
\end{array}\right] .
$$

The common set $\Gamma(\delta)$ of positive invariance is given by Figure 5.3 together with the sets $\mathscr{D}_{j}$ and $\Psi\left(P_{j}, \rho_{j}\right) ; \rho_{1}=8, \rho_{2}=9$.

The study of these two examples shows that the results of Theorem 4.5 are less conservative than the results of Theorem 4.7. This fact is due to the more constraining condition (4.32) which is needed with the use of a piecewise Lyapunov function candidate. Consequently, a common Lyapunov function, when it exists, is more adequate to the design of fuzzy controllers for a nonlinear system with constrained control. 


\section{Conclusion}

In this paper, the problem of constrained nonlinear systems represented by fuzzy systems is studied. The positive invariance tool is used. Sufficient conditions of asymptotic stability are obtained despite the presence of saturations on the control by using a common Lyapunov function and a piecewise Lyapunov function successively. The used approach is the one followed in $[8,9]$ with uncertain-like subsystems and upper bound subsystems. The obtained results are successfully applied to two nonlinear systems with constrained control, represented by Takagi-Sugeno-(TS-) type fuzzy model. This leads to the characterization of a symmetric ellipsoid and a polyhedral common region of positive invariance and asymptotic stability successively. It is also shown that a common Lyapunov function, when it exists, leads to less conservative region of positive invariance and asymptotic stability when the system is with constrained control.

\section{References}

[1] S. Bentalba and A. El Hajjaji, Parking controller design for vehicle dynamics using fuzzy logic, Journal on Automatic Control 40 (1999), no. 2, 26-29.

[2] A. Benzaouia, The resolution of equation $X A+X B X=H X$ and the pole assignment problem, IEEE Transactions on Automatic Control 39 (1994), no. 10, 2091-2095.

[3] __ Further results on the saturated controller design for linear continuous-time systems, Proceedings of the 10th Mediterranean Conference on Control and Automation (MED '02), Lisbon, July 2002.

[4] A. Benzaouia and C. Burgat, Regulator problem for linear discrete-time systems with nonsymmetrical constrained control, International Journal of Control 48 (1988), no. 6, 2441-2451.

[5] A. Benzaouia, A. El Hajjaji, and M. Naib, Stabilization of a class of constrained non linear systems by fuzzy control, To appear in International Journal of Innovative Computing, Information and Control, (2006).

[6] A. Benzaouia and A. Hmamed, Regulator problem for linear continuous-time systems with nonsymmetrical constrained control, IEEE Transactions on Automatic Control 38 (1993), no. 10, $1556-1560$.

[7] F. Blanchini, Set invariance in control, Automatica 35 (1999), no. 11, 1747-1767.

[8] S. G. Cao, N. W. Rees, and G. Feng, Stability analysis and design for a class of continuous-time fuzzy control systems, International Journal of Control 64 (1996), no. 6, 1069-1087.

[9] _ Analysis and design for a class of complex control systems. II. Fuzzy controller design, Automatica 33 (1997), no. 6, 1029-1039.

[10] C. L. Chen, P. C. Chen, and C. K. Chen, Analysis and design of fuzzy control systems, Fuzzy Sets and Systems 57 (1993), no. 2, 125-140.

[11] P. O. Gutman and P. Hagander, A new design of constrained controllers for linear systems, IEEE Transactions on Automatic Control 30 (1985), no. 1, 22-33.

[12] H. Hindi and S. Boyd, Analysis of linear systems with saturating using convex optimisation, Proceedings of 37th IEEE Conference on Decision Control, Florida, 1998, pp. 903-908.

[13] M. Johansson, A. Rantzer, and K. E. Arzen, Piecewise quadratic stability of fuzzy systems, IEEE Transactions on Fuzzy Systems 7 (1999), no. 6, 713-722.

[14] F. Khaber, K. Zehar, and A. Hamzaoui, State feedback controller design via takagi-sugeno fuzzy model: LMI approach, International Journal of Computational Intelligence 2 (2005), no. 2.

[15] K. Tanaka and M. Sano, Fuzzy stability criterion of a class of nonlinear systems, International Journal of Information Sciences 71 (1993), no. 1-2, 3-26. 
[16] _ A robust stabilization problem of fuzzy control systems and its application to backing up control of a truck-trailer, IEEE Transactions on Fuzzy Systems 2 (1994), no. 2, 119-134.

[17] K. Tanaka and M. Sugeno, Stability analysis and design of fuzzy control systems, Fuzzy Sets and Systems 45 (1992), no. 2, 135-156.

[18] H. O. Wang, K. Tanaka, and M. F. Griffin, An approach to fuzzy control of nonlinear systems: stability and design issues, IEEE Transactions on Fuzzy Systems 4 (1996), no. 1, 14-23.

A. El Hajjaji: Centre de Robotique, d'Electrotechnique, et d'Automatique, Université de Picardie, 7 Rue du Moulin Neuf, Picardie, 80000 Amiens, France E-mail address: ahmed.hajjaji@sc.u-picardie.fr

A. Benzaouia: Equipe d'Automatique et de Contrôle des Procédés Industriels, Departement Physique, Faculte des Sciences Semlalia Marrakech, L’Université Cadi Ayyad, BP 2390, Marrakech 40001, Morocco

E-mail address: benzaouia@ucam.ac.ma

M. Naib: Equipe d'Automatique et de Contrôle des Procédés Industriels, Departement Physique, Faculte des Sciences Semlalia Marrakech, L’Université Cadi Ayyad, BP 2390,

Marrakech 40001, Morocco

E-mail address: m.naib@ucam.ac.ma 


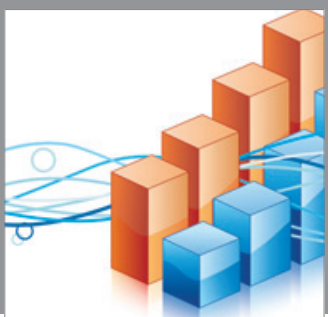

Advances in

Operations Research

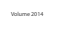

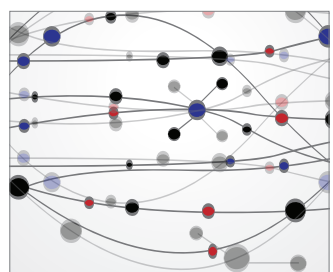

\section{The Scientific} World Journal
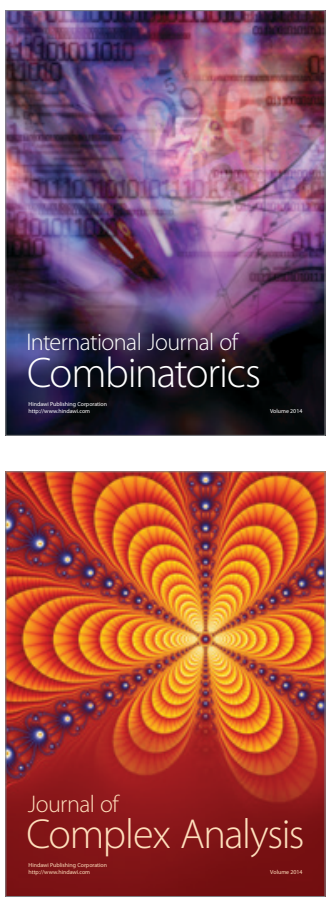

International Journal of

Mathematics and

Mathematical

Sciences
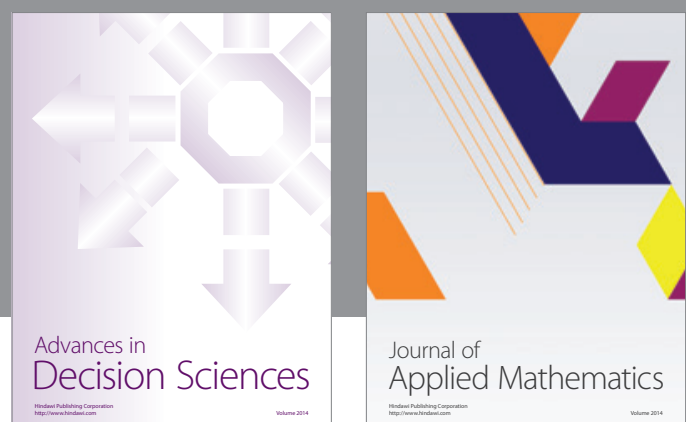

Journal of

Applied Mathematics
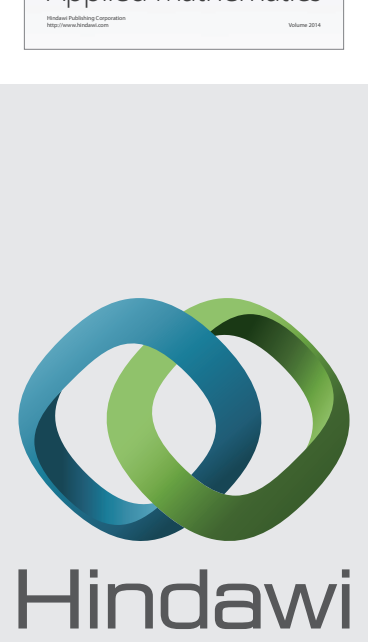

Submit your manuscripts at http://www.hindawi.com
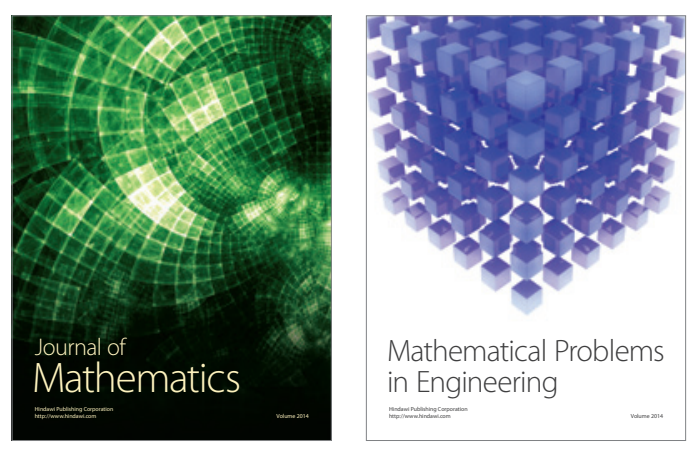

Mathematical Problems in Engineering
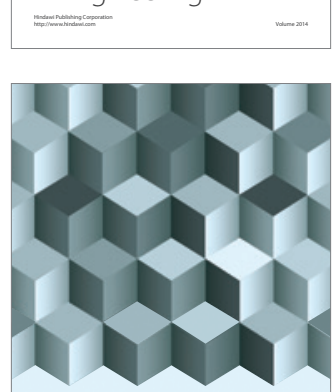

Journal of

Function Spaces
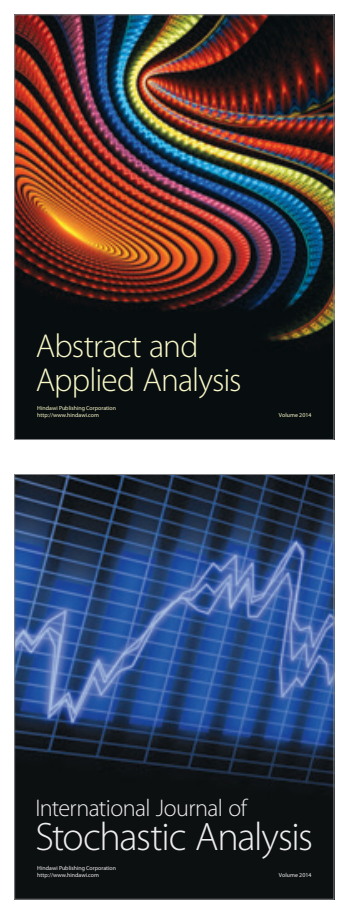

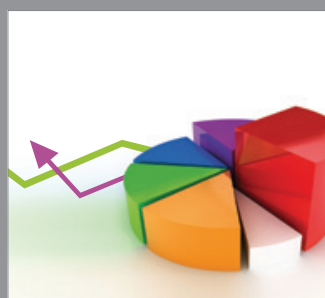

ournal of

Probability and Statistics

Promensencen
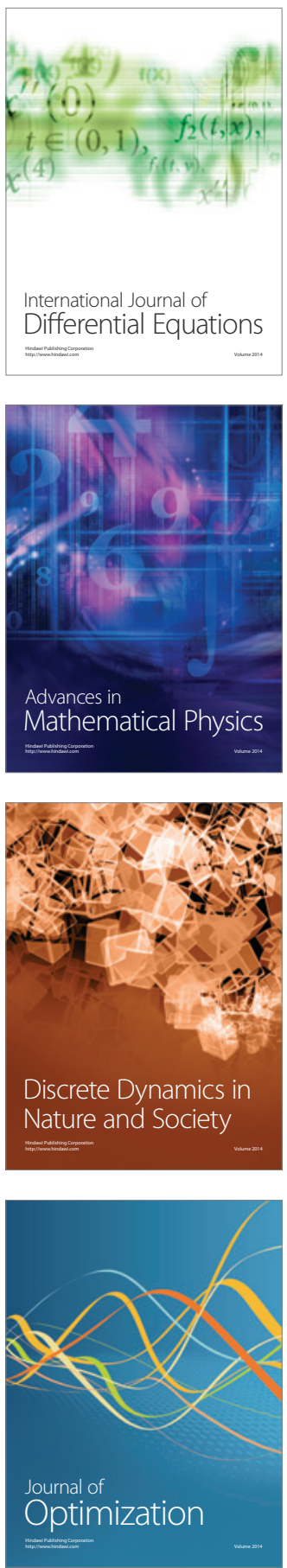